\title{
Carrier Transport in Ultra-Thin Nano/Polycrystalline Silicon Films and Nanowires
}

Toshio Kamiya ${ }^{1,2,4}$, Yong T. Tan ${ }^{2,4}$, Yoshikazu Furuta ${ }^{3,4}$, Hiroshi Mizuta ${ }^{3,4}$, Zahid A.K. Durrani ${ }^{2,4}$ and Haroon Ahmed ${ }^{2,4}$

${ }^{1}$ Materials and Structure Laboratory, Tokyo Institute of Technology, Japan

${ }^{2}$ Microelectronics Research Centre, Cavendish Laboratory, University of Cambridge, UK

${ }^{3}$ Hitachi-Cambridge Laboratory, UK

${ }^{4}$ CREST, JST, Japan

\begin{abstract}
Carrier transport was investigated in two different types of ultra-thin silicon films, polycrystalline silicon (poly-Si) films with large grains $>20 \mathrm{~nm}$ in size and hydrogenated nanocrystalline silicon (nc-Si:H) films with grains $4 \mathrm{~nm}-8 \mathrm{~nm}$ in size. It was found that there were local non-uniformities in grain boundary potential barriers in both types of films. Single-electron charging effects were observed in $30 \mathrm{~nm} \times 30 \mathrm{~nm}$ nanowires fabricated in 30 nm-thick nc-Si:H films, where the electrons were confined in crystalline silicon grains encapsulated by amorphous silicon. In contrast, the poly-Si nanowires of similar dimensions showed thermionic emission over the grain boundary potential barriers formed by carrier trapping in grain boundary defects.
\end{abstract}

\section{INTRODUCTION}

Recently, there has been considerable interest in the low temperature growth of hydrogenated microcrystalline silicon $(\mu \mathrm{c}-\mathrm{Si}: \mathrm{H})$ thin films for their potential applications in thin film solar cells and thin film transistors for flat panel displays [1,2]. Plasma-enhanced chemical vapor deposition (PECVD) is a novel technique that allows the film microstructure to be controlled by selecting appropriate deposition conditions [3]. For example, it is possible to prepare small grain nanocrystalline silicon (nc-Si:H) using a large $\mathrm{H}_{2} / \mathrm{SiF}_{4}$ gas mixing ratio. This controllability of grain size is promising for novel devices utilizing quantum confinement and single-electron charging effects.

It is difficult to fabricate very thin $(<50 \mathrm{~nm}) \mathrm{nc}-\mathrm{Si}: \mathrm{H}$ films with high crystallinity, large carrier mobility and large conductance using a low-temperature PECVD process. In addition, the transport properties of these heterogeneous materials are affected significantly by local microstructures. Thus carrier transport has been investigated in localized regions using an AFM/STM [4] and by fabricating wires ranging $0.1 \mu \mathrm{m}-4 \mu \mathrm{m}$ in width [5]. In this paper, we report the fabrication of very thin nc-Si:H films and their characterization using 30-nm-wide nanowires to investigate the local carrier transport properties. We compare these results with those 
obtained for non-hydrogenated, large grain polycrystalline silicon (poly-Si) films.

\section{EXPERIMENTAL}

Two different types of films highly doped with phosphorus were used. The first was a 50 nm-thick poly-Si film prepared by solid-phase-crystallization (SPC). It was thermally annealed at $850^{\circ} \mathrm{C}$ for $30 \mathrm{~min}$ after phosphorus ion implantation (see ref. 6). The other was a nc-Si:H film deposited by $100 \mathrm{MHz}$-very high frequency PECVD from a SiF $4: \mathrm{H}_{2}: \mathrm{SiH}_{4}: \mathrm{PH}_{3}$ gas mixture (see ref. 3). The flow rates of the $\mathrm{SiF}_{4}, \mathrm{H}_{2}$ and $\mathrm{SiH}_{4}$ were 30, 40 and $0.25 \mathrm{sccm}$, respectively. The concentration of $\mathrm{PH}_{3}$ in $\mathrm{SiH}_{4}$ was $2 \%$. The film thickness varied from $10 \mathrm{~nm}$ to $1 \mu \mathrm{m}$ in this case. The films were deposited either on Corning 7059 glass or on a 150-nm-thick silicon oxide layer grown thermally on top of crystalline silicon.

Double side-gated very short nanowire (NW) structures were defined using electron-beam lithography (see ref. 7). The width and length of the NWs were $30 \mathrm{~nm}$ and $30-40 \mathrm{~nm}$ respectively. Figure 1 shows a scanning electron microscope (SEM) image of a typical NW structure.

We have characterized the structure of the nc-Si:H films using reflective high energy electron diffraction (RHEED) and transmission electron microscopy (TEM). Impurity concentrations were measured by secondary ion mass spectroscopy. Electrical characteristics were measured at temperatures ranging from $25 \mathrm{~K}$ to $300 \mathrm{~K}$. The conductivity of the films was measured using transmission-line test structure electrodes of $1.5 \mathrm{~mm}$ in width and $0.2 \mu \mathrm{m}-4.0 \mu \mathrm{m}$ in length. Hall effect measurements were performed to evaluate carrier mobility and concentration.

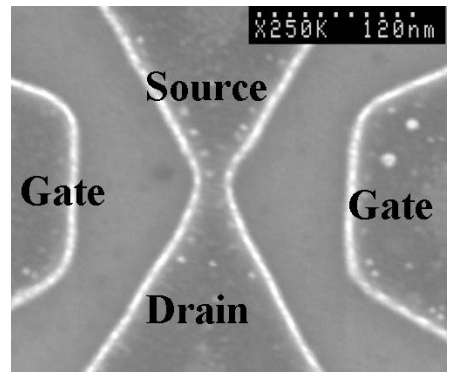

Fig. 1 SEM image of a Fig. 2 RHEED images of double side-gated NW. 10-nm-thick nc-Si:H film. Channel dimension is $30 \mathrm{~nm}$ wide and $40 \mathrm{~nm}$ long.
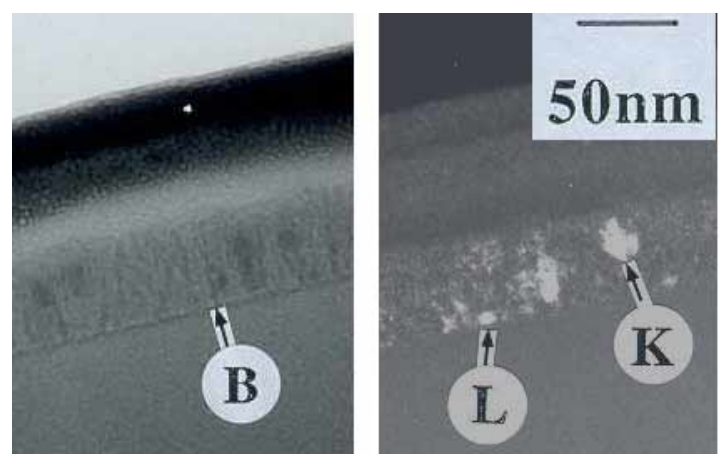

Fig. 3 TEM images of a $50 \mathrm{~nm}$-thick nc-Si:H film. Bright field image using Fresnel contrast (left) and dark field image (right). " $\mathrm{B}$ " indicates a typical grain boundary.

\section{RESULTS AND DISCUSSIONS}

\section{Film structure}

We have reported previously the film microstructure for the poly-Si film [7]. The Raman spectra showed a sharp peak corresponding to crystalline silicon grains at $\sim 520 \mathrm{~cm}^{-1}$ and a broad peak at 
$\sim 500 \mathrm{~cm}^{-1}$. The latter is attributed to a disordered phase such as grain boundaries and amorphous silicon. The volume fraction of the disordered phase is estimated to be $\sim 14 \%$ using the peak-area ratio. The grain size ranged from $20 \mathrm{~nm}$ to $150 \mathrm{~nm}$, measured by SEM using a Secco etched sample. Phosphorus-concentration was $1.2 \times 10^{19} / \mathrm{cm}^{3}$ at the film surface and decreased to $3.5 \times 10^{18} / \mathrm{cm}^{3}$ at the substrate interface. It is thought that the ion-implantation and the SPC processes caused the non-uniform P-concentration distribution.

For the nc-Si:H films, we can see sharp diffraction rings superimposed on a broad background in a RHEED image even in the 10-nm-thick film (Fig. 2). This indicates that the film contains crystalline silicon grains embedded in amorphous silicon ( $\mathrm{a}-\mathrm{Si}: \mathrm{H})$ tissues. In a bright-field TEM image [Fig. 3(a)], we observed large, corn-shaped structures with lateral size $\sim 30 \mathrm{~nm}$ at the film surface. The dark-field image [Fig. 3(b)] shows a high contrast for the crystalline grains. It indicates that each crystalline grain ("K" and " $L$ " in the figure) is spherical and smaller than the large corn-shaped structure. This type of secondary microstructure feature is often observed in $\mu \mathrm{c}-\mathrm{Si}: \mathrm{H}[8,9]$. From high resolution TEM, we confirmed that the smaller structures corresponded to single-domain crystalline silicon grains $4 \mathrm{~nm}-8 \mathrm{~nm}$ in size. Crystalline fractions and carrier concentrations are evaluated to be $\sim 60 \%$ and $1.1 \times 10^{20} / \mathrm{cm}^{3}$ respectively for the 25 -nm-thick film and $\sim 70 \%$ and $3.7 \times 10^{20} / \mathrm{cm}^{3}$ respectively for the 50 -nm-thick film. Since the P-concentration was measured to be $4.8 \times 10^{20} / \mathrm{cm}^{3}$, the dopant activation efficiency corresponded to $77 \%$ for the 50-nm-thick film.

The above results characterize clearly the specific features of these films. The poly-Si film has rather large crystalline grains $>20 \mathrm{~nm}$ in size. In contrast, the $\mathrm{nc}-\mathrm{Si}: \mathrm{H}$ film has much smaller single-domain grains 4-8 $\mathrm{nm}$ in size and contains a larger amorphous content.

\section{Carrier transport in films and very short nanowires}

Figure 4 shows the temperature dependence of conductivity for the films and the NWs. The carrier mobility measured by Hall measurements is $\sim 1.8 \mathrm{~cm}^{2} / \mathrm{Vs}$ for the 50 -nm-thick nc-Si:H films. In contrast, larger mobility of $\sim 70 \mathrm{~cm}^{2} / \mathrm{Vs}$ is obtained for the poly-Si films. The larger mobility can be attributed to the larger grain size and the smaller amorphous content in the poly-Si films in part. We evaluate the activation energy of the conductivity $\left(E_{\mathrm{a}}\right)$ from the slope of the Arrhenius plot at around room temperature because the conductivity shows percolation behavior [10]. The $E_{\mathrm{a}}$ of the nc-Si:H films depends largely on the film thickness. Thinner films will have a larger activation energy. This is thought to be due to the destruction of carrier percolation paths, as discussed later.

For NWs, the absolute values of the conductivity are much less than in the bulk film. It is thought that the low apparent conductivity in the NWs is due to geometrical effects: i.e. carriers are scattered at the device surface in and near the narrow channel regions.

In contrast to the macroscopic measurements of $E_{\mathrm{a}}$ values in bulk films, we expect to obtain a 
large variation in the $E_{\mathrm{a}}$ values in NWs. This may be attributed to a statistical distribution of grain boundary properties. As we are investigating local transport properties in the nanoscale regime, we can expect significant variations in the $E_{\mathrm{a}}$ values. Thus, the $E_{\mathrm{a}}$ values obtained for the NWs reflect the local potential barrier height at grain boundaries. Indeed we have found that the $E_{\mathrm{a}}$ values, which corresponds to the potential barrier height, ranged from $7 \mathrm{meV}$ to $80 \mathrm{meV}$ in the poly-Si film. Variation is also seen in the nc-Si:H film, where different $E_{\mathrm{a}}$ values are observed with a maximum value of $40 \mathrm{meV}$ [see Fig. 4(b)], however, the variation is smaller than in the poly-Si film.
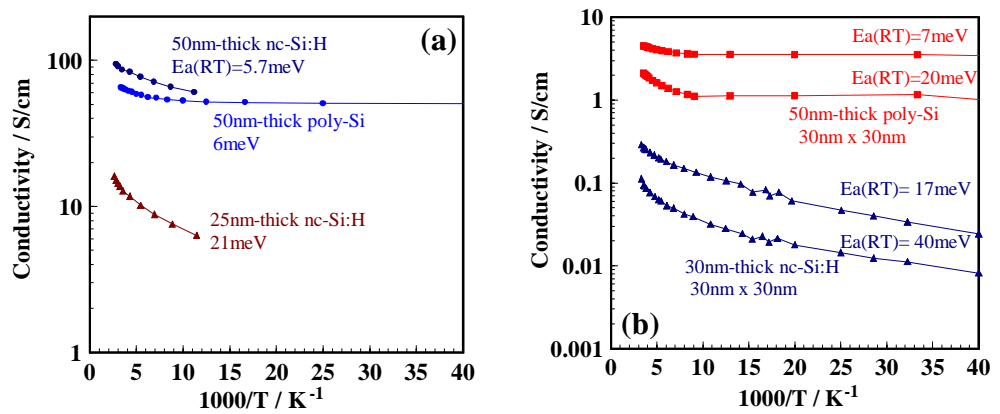

Fig. 4 Temperature dependence of conductivity for the films measured with coplanar electrodes (a) and for NWs (b). Data obtained for two different NWs are shown in figure (b) for each film. The numbers indicate the room temperature activation energy and the film thickness.

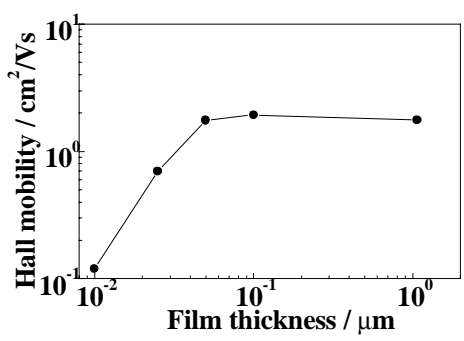

Fig. 5 Film thickness dependence of Hall mobility.
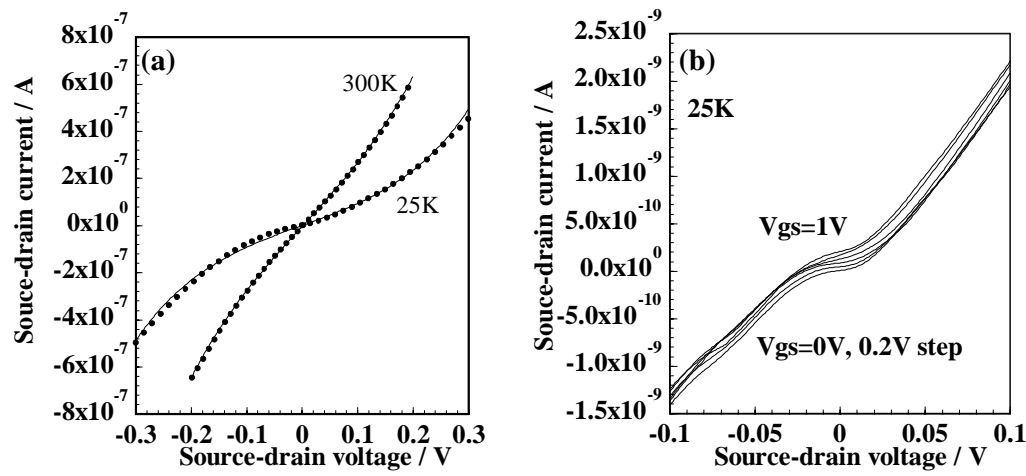

Fig. $6 \mathrm{I}_{\mathrm{ds}}-\mathrm{V}_{\mathrm{ds}}$ characteristics measured for poly-Si (a) and nc-Si:H (b). Numbers in the figures indicate temperature.

Figure 5 shows the electron Hall mobility plotted against the thickness of the nc-Si:H film. As film thickness increases the Hall mobility increases monotonically and at a thickness $>50 \mathrm{~nm}$ it saturates. Since we have observed a decrease in the activation energy of the mobility when the film thickness exceeded $50 \mathrm{~nm}$, we speculate that carrier transport percolation paths are formed when film thickness reaches $\sim 50 \mathrm{~nm}$. The percolation paths are thought to be through crystalline grains. The results suggest that some crystalline grains are surrounded by a-Si:H for film thicknesses less than $50 \mathrm{~nm}$. It is noteworthy that the mobility behavior obtained for the nc-Si:H films is different from that we previously reported for preferentially oriented poly-Si:H films, which is related to the different microstructure evolution [11]. 
When we measured the source-to-drain current-voltage (I-V) characteristics of 30-nm-wide and 40-nm-long NWs fabricated in the 50-nm-thick poly-Si films, 30\% of the poly-Si NWs exhibited non-linear I-V characteristics. Dots in Fig. 6(a) show the I-V characteristics of a typical poly-Si NW measured at $300 \mathrm{~K}$ and $25 \mathrm{~K}$. The solid lines show the results fitted to eq. (1) [12].

$$
I(V)=2 q n_{P}\left(\frac{k_{B} T}{2 \pi m^{*}}\right)^{1 / 2} \exp \left(-\frac{q V_{B}}{k_{B} T}\right) \sinh \left(-\frac{q V}{2 n k_{B} T}\right) .
$$

$q$ : carrier charge, $m^{*}$ : effective mass, $k_{\mathrm{B}}$ : Boltzmann constant, $V_{\mathrm{B}}$ : barrier height $T$ : temperature, $n$ : number of grain boundaries, $V:$ applied voltage

We found that the measured I-V characteristics agreed well with eq. (1) at temperatures greater than $120 \mathrm{~K}$, supporting the idea that the carrier transport proceeds by means of thermionic emission over grain boundary potential barriers [12]. At lower temperatures, we can see a significant deviation between the data measured and fitted. As the conductivity is almost independent of temperature in this temperature range, as seen in Fig. 4, we attribute the carrier transport to tunnelling or variable-range-hopping conduction mechanisms. Since the poly-Si film is heavily doped and the grain boundary defects are not passivated, it is likely that the carriers flow through the $\mathrm{P}$ levels and/or grain boundary defect states. The results are consistent with the idea that double Schottky barriers are formed at the grain boundaries due to carrier trapping in defect states. Individual grain boundaries may have different defect density related to different grain size, orientation and boundary thickness. It would cause the distribution in the potential height.

For the $30 \mathrm{~nm}$ thick $\times 30 \mathrm{~nm}$ wide $\times 30 \mathrm{~nm}$ long nc-Si:H NWs, $70 \%$ of devices exhibited linear I-V characteristics while other devices show non-linear I-V characteristics [see Fig. 6(b)]. The I-V characteristic is fully modulated by the gate-bias $\left(\mathrm{V}_{\mathrm{gs}}\right)$ and we observed clear gate-bias conductance oscillations. This is evidence of Coulomb blockade effects. It is thought that the nanocrystalline silicon grains behave as charging islands and the amorphous silicon layer surrounding the crystalline grains behave as tunnelling barriers [10]. The distribution in the potential barrier height seen in Fig. 4 (b) would be due to the statistical fluctuation in the Fermi level in the a-Si:H layers because one a-Si:H grain boundary has only several phosphorus atoms.

For nc-Si:H NWs longer than $1 \mu \mathrm{m}$, we observed Coulomb blockade effects in all NWs. This is because the longer wires have a higher probability of containing charging islands encapsulated by good a-Si:H tunnelling barriers and multiple tunnel junctions. In contrast to the poly-Si NWs, we did not observe thermionic emission behavior in any nc-Si:H NW at high temperature. To observe I-V non-linearities, $q V / 2 n k_{\mathrm{B}} T$ in eq. (1) must be large. Since the grain size in the nc-Si:H film is much smaller than that in the poly-Si film, the applied voltage used would be too low to observe thermionic emission behavior. Alternatively, since the grain boundaries are passivated with hydrogen and some grains are covered with a-Si:H, we may need to consider different carrier 
transport mechanisms through these grain boundaries.

\section{CONCLUSIONS}

Using PECVD and a fluorinated source gas, we can fabricate very thin $(>10 \mathrm{~nm}) \mathrm{nc}-\mathrm{Si}: \mathrm{H}$ films on a-SiO${ }_{x}$ at $300^{\circ} \mathrm{C}$. We have compared the carrier transport in the nc-Si:H films with transport in larger grained poly-Si films. The grain boundary potential height is found to be $<80 \mathrm{meV}$ in the poly-Si film and $<40 \mathrm{meV}$ in the nc-Si:H film. Nc-Si:H NWs, $30 \mathrm{~nm} \times 30 \mathrm{~nm} \times 30 \mathrm{~nm}$ in size, exhibit single-electron charging effects with the electrons confined in the crystalline grains and a-Si:H forming tunnel barriers. In the poly-Si film we observe thermionic emission over grain boundary potential barriers. It is thought that the potential barrier is formed due to carrier trapping in grain boundary defects in the non-hydrogenated poly-Si film.

\section{ACKNOWLEDGEMENTS}

This work is supported by the Japan Science and Technology through the Core Research for Evolutional Science and Technology (CREST) program.

\section{REFERENCES}

1. J. Meier, S. Dubail, J. Cuperus, et al., J. Non-Cryst. Sol. 227-230, 1250 (1998).

2. T. Sameshima, Applied Surface Science 96-98, 352 (1996).

3. T. Kamiya, K. Nakahata, C.M. Fortmann and I. Shimizu, J. Non-Cryst. Sol. 266-269, 120 (2000).

4. A. Fejfar, B. Rezek, P. Knapek, J. Stuchlik and J. Kocka, J. Non-Cryst. Sol. 266-269, 309 (2000).

5. J. W. Tringe and J.D. Plummer: J. Appl. Phys. 49, 7913 (2000).

6. Y. Furuta, H. Mizuta, K. Nakazato, Y.-T. Tan, T. Kamiya, Z.A.K. Durrani, H. Ahmed and K. Taniguchi, Jpn. J. Appl. Phys. Lett. (2001) accepted.

7. Y.T. Tan, Z.A.K. Durrani and H. Ahmed, J. Appl. Phys. 89, 1262 (2001).

8. T. Kamiya, K. Nakahata, K. Ro, C.M. Fortmann and I. Shimizu, Jpn. J. Appl. Phys. 38, 5750 (1999).

9. E. Vallat-Sauvain, U. Kroll, J. Meier, N. Wyrsch and A. Shah, J. Non-Cryst. Sol. 266-269, 125 (2000).

10. Y.T. Tan, T. Kamiya, Z.A.K. Durrani and H. Ahmed, Appl. Phys. Lett. 78, 1083 (2001).

11. T. Kamiya, K. Nakahata, Y.T. Tan, Z.A.K. Durrani and I. Shimizu, J. Appl. Phys. (June, 2001) in print.

12. T. Kamins, Polycrystalline silicon for integrated circuits and display, 2nd ed (Kluwer, Boston, 1998). 